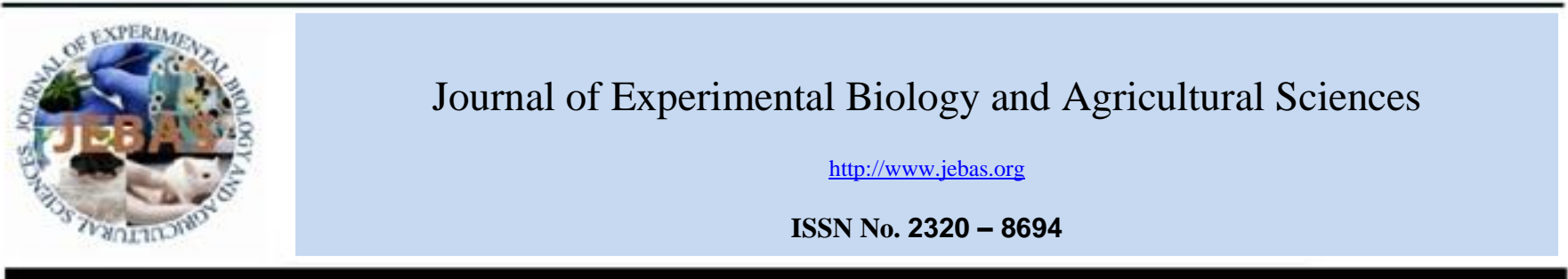

\title{
SPECTRAL COMPOSITION OF LIGHT AS A FACTOR IN THE REGULATION OF PLANT METABOLISM
}

\section{Tatiana Petrovna Iakushenkova* (iD), Dalal Al-Hussein (ic)}

Department of Botany and Plant Physiology, Institute of Fundamental Medicine and Biology, Kazan Federal University, Russia, Kazan, Kremlyovskaya St., 18 Kazan, Respublika Tatarstan, Russia, 420008

Received - September 25, 2020; Revision - November 14, 2020; Accepted - December 08, 2020

Available Online December 15, 2020

DOI: http://dx.doi.org/ 10.18006/2020.8(Spl-2-AABAS).S308.S313

\section{KEYWORDS \\ Kale cabbage \\ Curled leaf parsley \\ Lettuce \\ Protein \\ Provitamin A ascorbic acid \\ Carotenoids antioxidant activity \\ Spectral composition of light}

\begin{abstract}
Plants have many evolutionary developed adaptation mechanisms to ensure functional flexibility under the influence of environmental factors, including the synthesis of secondary metabolites that perform various functions in response to environmental changes, growth, and development. Further, these changes can be caused by various environmental factors, which include local geo-climatic, seasonal changes, temperature conditions, lighting, and humidity. All of them affect the accumulation of biomass and the biosynthesis of primary and secondary plant metabolites. The formation of secondary metabolites by plants is considered their adaptive ability to cope with the stressful conditions of a changing environment. Within the framework of this study, the biochemical status of Brassica oleracea var. Sabellica, Lactuca sativa, Petroselinum crispum plants were determined when grown indoors under various light spectral composition. To determine the biochemical status, the plants were analyzed for the content of ascorbic acid, provitamin A, carotenoids and proteins, and their antioxidant activity was also determined. Results of the study revealed that the blue part of the light spectrum induces the accumulation of ascorbic acid, provitamin A, carotenoids and protein in all studied species. Further, it was also reported that Kale cabbage has the highest accumulation of ascorbic acid, proteins, carotenoids, and also has the highest antioxidant activity as compared to the other studied species.
\end{abstract}

* Corresponding author

E-mail: tyakushekfu@gmail.com (Tatiana Petrovna Iakushenkova)

Peer review under responsibility of Journal of Experimental Biology and Agricultural Sciences.

Production and Hosting by Horizon Publisher India [HPI] (http://www.horizonpublisherindia.in/).

All rights reserved.
All the articles published by Journal of Experimental Biology and Agricultural Sciences are licensed under a Creative Commons Attribution-NonCommercial 4.0 International License Based on a work at www.jebas.org.

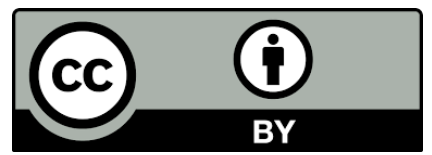




\section{Introduction}

Solar radiation absorbed by plants is one of the fundamental conditions for their existence since it acts as a source of energy, which, as we know, is used by plants in photosynthesis. The artificial light environment in phytotrons and greenhouses is inherently very different compared to natural light. This reflects its effect on many aspects of plant functioning, including photosynthesis, photomorphogenesis, water exchange, mineral nutrition, biomass, quantity, and quality of the crop. For indoor growing plants, metal halide lamps, highpressure sodium lamps (HPS) and/or fluorescent lamps are used in most cases, either as an additional source or as a sole source. They are often ineffective for growing plants compared to sunlight (Morrow, 2008; Darko et al., 2014). Plants grown under these artificial lighting conditions often show many symptoms of sub-optimal lighting conditions, such as elongation of internodes, altered flowering times, and poor quality and quantity of crop production. Besides, these lamps have high power consumption, and high operating temperatures make them economically disadvantageous (Morrow, 2008).

The search for new plant species and varieties with high primary and secondary metabolites content related to human health is the primary task of any crop production program. To provide necessary vitamins, antioxidants, proteins, and mineral elements to the growing population many types of plants are cultivated in greenhouses all year round. When plants are growing indoors light is often the most limiting factor. The intensity and spectral composition are two main characteristics of light that must be taken into account to create the most favorable conditions for the growth of a particular plant species.

Currently, plant physiologists pay special attention to the study of kale or Kale cabbage (Lat. Brassica oleracea var. Sabellica), which is a specific variety of white cabbage. It is a rich source of various vitamin $\mathrm{A}, \mathrm{C}, \mathrm{E}, \mathrm{K}, \mathrm{PP}$, and vitamin B complex (thiamine, riboflavin, pantothenic acid). Along with this leaves of this plant has high contents of various pigments and mineral such as betacarotene, carotenoids, lutein, zeaxanthin, and minerals such as potassium, calcium, sodium, magnesium, phosphorus (Morrow, 2008; Kaulmann et al., 2014; Walsh et al., 2015). It contains a large amount of vitamin $\mathrm{K}$ which is several times higher than the daily intake (DI). Further, raw cabbage has $84 \%$ water, $9 \%$ carbohydrates, $4 \%$ protein, and $1 \%$ fat. Like broccoli and other cruciferous vegetables, kale cabbage contains glucosinolate compounds such as glucoraphanin, which promotes the formation of sulforaphane, a compound that helps to prevent cancer (Houghton et al., 2013). Cabbage Kale is also rich in polyphenols such as ferulic acid which has antioxidant properties (Korus \& Lisiewska, 2011).
Curled leaf parsley (Petroselinum crispum) is a biennial plant growing in temperate climates or an annual herb of subtropical and tropical regions. It is a good source of various flavonoids and antioxidants such as luteolin, apigenin, folic acid, vitamin $\mathrm{K}$, vitamin C, and vitamin A (Meyer et al., 2006). One gram of dried parsley leaves contains about $6.0 \mathrm{mg}$ of lycopene and $10.7 \mathrm{mg}$ of alpha-carotene, $82.9 \mathrm{mg}$ lutein + zeaxanthin, and $80.7 \mathrm{mg}$ betacarotene.

Lettuce leaves (Lactuca sativa) is an annual leafy vegetable and are an excellent source of vitamin A and $\beta$-carotenoids. It's a lowcalorie food and $100 \mathrm{~g}$ of fresh leaves provide only 15 calories of energy. However, it contains many phytonutrients, which help in improve health and prevent many diseases. Fresh lettuce leaves are an excellent source of vitamins $\mathrm{A}$ and $\beta$-carotenes and $100 \mathrm{~g}$ of fresh, raw lettuce provides $247 \%$ of the daily intake value of vitamin A and 4,443 mcg of carotene. Antioxidant properties of this plant are well reported and this might be because of these two compounds. Fresh lettuce leaves have a higher amount of folate and vitamin C. Folate is part of the co-factors in the metabolism of enzymes required for DNA synthesis and therefore plays a vital role in the prevention of neural tube defects in the fetus during pregnancy. It is also a rich source of vitamin B complex such as thiamine, vitamin B-6 (pyridoxine), riboflavin. Regular inclusion of lettuce leaves in the diet prevents osteoporosis, iron deficiency anemia, and protect the body from cardiovascular disease, ARMD, Alzheimer's disease and cancer. The current study aimed to access to effect of light spectrum composition on the metabolism of three selected plant species.

\section{Materials and Methods}

\subsection{Light spectrum}

For growing plants, a phytotron was used which divided the light spectrum into three light-insulated blocks that have different light sources. Three different wavelength fluorescent lamps were used as light sources: WL 40 - white light (control), RL 40 - red light, and BL 40 - blue light.

\subsection{Determination of the content of ascorbic acid in plants}

Quantitative estimation of the ascorbic acid content in three selected plant species was carried out spectrophotometrically. In an acidic medium, ascorbic acid reduced potassium hexacyanoferrite $\left(\mathrm{Fe}^{+3}\right)$ to potassium hexacyanoferrate $\left(\mathrm{Fe}^{+2}\right)$, which forms iron hexacyanoferrate (Prussian blue) in the presence of ferric ions. Moreover, in the presence of fluorine ions in the medium, this Prussian blue does not precipitate and it remained blue.

For ascorbic acid estimation, 500mg of plant leaves sample was ground in $1 \mathrm{ml}$ of buffer solution ( $\mathrm{pH} 3.69$ ), and transferred into Eppendorf tubes for centrifugation which was carried out for $5 \mathrm{~min}$ 
at $12,500 \mathrm{~g}$. The supernatant (extract) was used for the ascorbic acid estimation which carried out by the following formula:

$\mathrm{C}=(\mathrm{K} \cdot \mathrm{V} \cdot \mathrm{X}) /(\mathrm{m} \cdot \Delta \mathrm{m} \cdot \mathrm{L})$

where:

$\mathrm{C}$ - content of ascorbic acid, $\mu \mathrm{g} / \mathrm{g}$ fresh weight,

$\mathrm{K}$ - concentration of ascorbic acid, $\mu \mathrm{g} / \mathrm{ml}$,

$\mathrm{V}$ - the total volume of the extract, $\mathrm{ml}$,

$\mathrm{X}$ - dilution of the extract in the reaction mixture (in this case, 6 times),

L - optical path length, cm,

$\mathrm{m}$ - weight of a wet sample, g,

$\Delta \mathrm{m}$ - the ratio of dry weight to wet weight (see Ch. 5.1.).

The measurements were carried out in triplicates and the standard error was calculated in Microsoft Office Excel (option "Descriptive statistics") (Sibgatullina et al., 2011).

\subsection{Determination of antioxidant activity}

Antioxidant activity of the selected plants was carried out by in-vitro oxidation of adrenaline which prevents the formation of reactive oxygen species (Sirota, 2000). For this, $0.2 \mathrm{ml}$ of $0.1 \%$ (5.46 mm) epinephrine hydrochloride solution was added to $3.4 \mathrm{ml}$ of $0.2 \mathrm{M}$ sodium carbonate buffer with $\mathrm{pH}$ 10.6. Prepared mixture mixed thoroughly and observations were taken by spectrophotometer (D1) at a wavelength of $347 \mathrm{~nm}$ in a $10 \mathrm{~mm}$ cuvette (Sirota, 2000). This was followed by adding $0.2 \mathrm{ml}$ of $01 \%$ epinephrine hydrochloride and $3.4 \mathrm{ml}$ of buffer $(\mathrm{pH}=10.6)$ in the previously prepared test solution, and OD was taken as described above (D2). To take into account the effect of the intrinsic color of extracts that absorb a certain wavelength in the visible part of the spectrum, a buffered extract solution without adrenaline was used as a control sample (Riabinina et al., 2011). The antioxidant activity of the studied samples was expressed as percent inhibition of adrenaline oxidation and this was calculated by the following formula:

$$
\mathrm{AA}=\frac{\left(\mathrm{D}_{1}-\mathrm{D}_{2}\right) * 100}{\mathrm{D}_{1}} \%
$$

\subsection{Lowry protein quantification}

The protein content was determined spectrophotometrically according to the Lowry method (Lowry et al., 1951). The method is based on the formation of a biuret complex, which in the presence of phenol, gives a characteristic color in a proportion of protein amount. For this, $200 \mathrm{mg}$ of the tested plant tissue was ground with $50 \mathrm{ml}$ of 8 percent phosphate buffer $(\mathrm{pH}$ 7.4) in a porcelain mortar until a homogeneous mass (homogenate) formed, and protein was extracted for $1 \mathrm{~h}$ with constant stirring at $4{ }^{\circ} \mathrm{C}$. This homogenized mixture was centrifuged for $10 \mathrm{~min}$ at $8000 \mathrm{~g}$ and the supernatant was used for protein concentration determination.
To determine the protein concentration in test samples, $1.25 \mathrm{ml}$ of reagent $\mathrm{C}$ was added to $0.25 \mathrm{ml}$ of the protein solution and mixed well for 5-10 minutes. This was followed by the addition of 0.125 $\mathrm{ml}$ of reagent $\mathrm{D}$. The solution was thoroughly mixed and left for 20-30 min in the dark. Instead of a sample, a similar amount of buffer or extraction solution was taken as a reference solution. The optical density of the solution was measured at a wavelength of $600-750 \mathrm{~nm}$. The protein content in the sample in $\mathrm{mg} / \mathrm{l}$ was determined using the calibration curve (Lowry, 2005).

\subsection{Determination of carotenoid content}

For carotenoid content determination, the pigments were extracted with $85 \%$ acetone and the absorbance of the extracts was measured on a Unico 2800 UV/VIS spectrophotometer at 663 and $664 \mathrm{~nm}$ for chlorophylls and $452.5 \mathrm{~nm}$ for carotenoids. The pigment concentration was calculated by using the equations: For $85 \%$ acetone (Rebelen):

C chl. $\mathrm{a}+$ chl. $\mathrm{b}=6.4 \mathrm{D}_{663}+18.8 \mathrm{D}_{664}$

$\mathrm{C}$ car $=4.75 \mathrm{D}_{452.5}-0.226 \mathrm{C}$ chl. $\mathrm{a}+$ chl. $\mathrm{b}$

Having determined the concentration of the pigment, we find its content in the experimental material by the formula:

$$
X=\frac{100 * B}{A}
$$

Where:

B - the amount of chlorophyll in the extract, mg;

A - the mass of fresh leaves taken for analysis, mg;

100 - coefficient for expression as a percentage.

\subsection{Statistical Analysis}

All experiments were carried out at least in triplicates. Statistical analysis of the data was carried out using standard mathematical methods using Microsoft Excel-2010 software (the arithmetic mean values and their standard errors were determined).

\section{Results and Discussion}

\subsection{The content of ascorbic acid in plants grown under a different spectrum of light}

Vitamins are a dietary supplement and involved in the functioning of all internal organs and systems. Without their constant intake, healthy life is impossible. Vitamins C plays an important role in the treatment of scurvy, tissue repair, enzyme functioning, antioxidant, and enhance the functioning of the immune system. It is a water-soluble vitamin, which means that it cannot be stored in the body, and we must constantly replenish the pool of this vitamin. Eating fruits and vegetables rich in vitamin $\mathrm{C}$ does not 


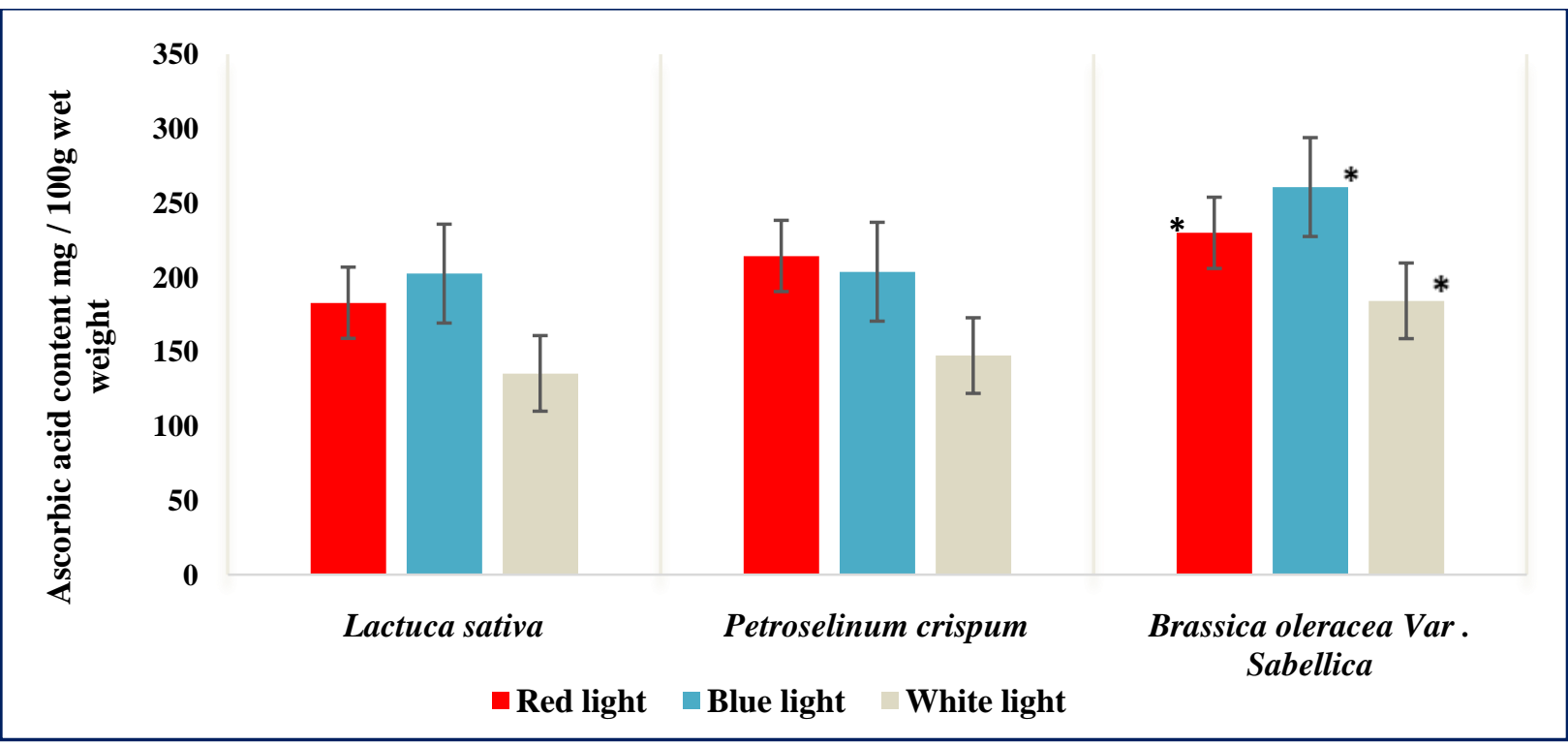

Figure 1 Effect of the light spectrum on the accumulation of ascorbic acid content in the studied plant species (Values indicated with asterisks are significantly different at $\mathrm{p} \leq 0.5$, each experiment was conducted in triplicates)

always meet the needs of our body, so the search for foods rich in vitamin $\mathrm{C}$ remains relevant to the present. In this regard, the current study investigated the content of ascorbic acid in the studied species when they were grown on different quality light spectrum (Figure 1).

Results of the study revealed an influence of light spectral composition on the accumulation of ascorbic acid and the highest accumulation of ascorbic acid was reported in cabbage Kale and this was followed by the parsley and lettuce leaves, regardless of the spectral composition of light. Further, among the tested three lights, the blue part of the spectrum had a positive effect on the accumulation of this vitamin in all studied species. While all the studied plant species grown under white light conditions had the least concentration of ascorbic acid. Further, Red light also promoted the accumulation of vitamin $\mathrm{C}$, but to a lesser extent than Blue light. Thus, the blue part of the spectrum in Kale cabbage contributed to the highest accumulation of this vitamin.

\subsection{Influence of the spectral composition of light on antioxidant activity}

Antioxidants are the substances that retard or prevent oxidation of biochemicals and extent the self-life of the oxidizable substance. Most of the diseases are mainly associated with the oxidative stress caused due to the accumulation of free radicals. In general, reactive oxygen species circulating in the body tend to react with electrons of other molecules, these can also affect the functioning of various enzyme systems and may cause or associated with various diseases or physiological disorder such as cancer, ischemia, aging, respiratory distress in adults, syndromes, rheumatoid arthritis, etc. A plant-based diet could protect the human body against various chronic, oxidative stress-related diseases. Many plants that are beneficial to health contain various chemical compounds that have antioxidant properties. It has been suggested that plant antioxidants may have positive effects on human health.

Analysis of the data related to the degree of adrenaline inhibition or autooxidation in the studied plants found that Kale cabbage reliably possesses greater antioxidant activity compared to other plant species. The leaves of Kale cabbage, grown in the blue part of the spectrum, had the highest antioxidant activity. The spectral composition of light did not have a significant effect on the antioxidant activity in lettuce and parsley (Figure 2).

\subsection{Effect of different light spectrum on the accumulation of proteins}

Proteins are complex organic compounds consisting of amino acids and are an important part of daily dietary intake. These proteins perform many vital functions related to the build or repair of tissue in the human body. These also play an important role as enzymes and hormones. Protein is an essential building block of bones, muscles, cartilage, skin, and blood, and forms compounds that provide immunity to protect the body from diseases.

Based on the data, it was found that the blue spectrum has a significant positive effect on the increase in the concentration of proteins in the species under study. Kale cabbage leaves contained the highest amount of protein compared to other plant species (Figure 3). 


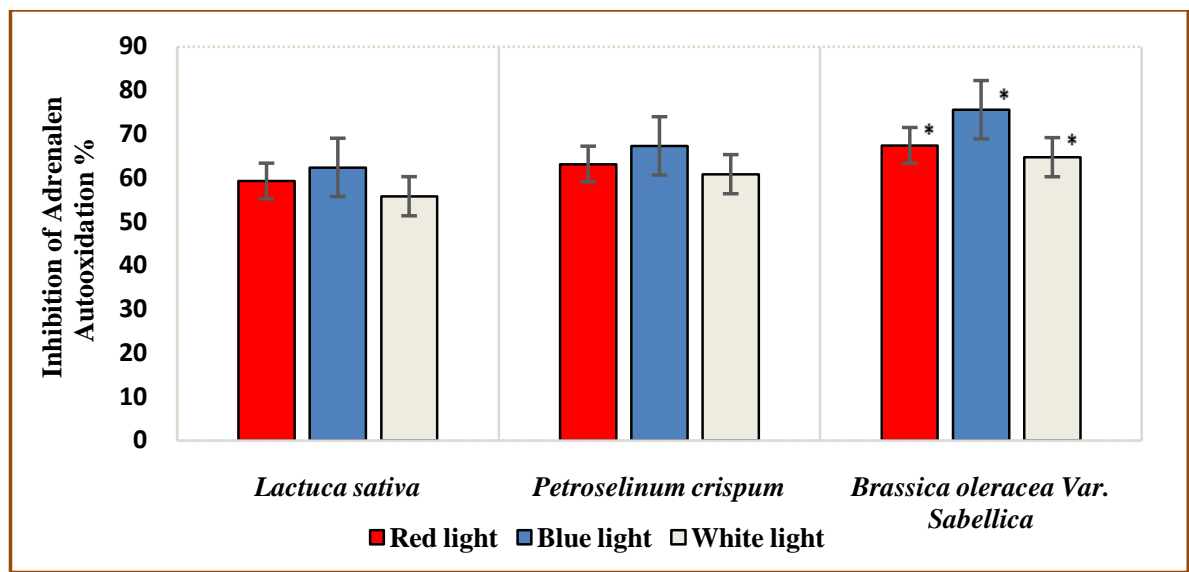

Figure 2 Effect of the light spectrum on the adrenaline inhibition properties of the studied plant species (Values indicated with asterisks are significantly different at $\mathrm{p} \leq 0.5$, each experiment was conducted in triplicates)

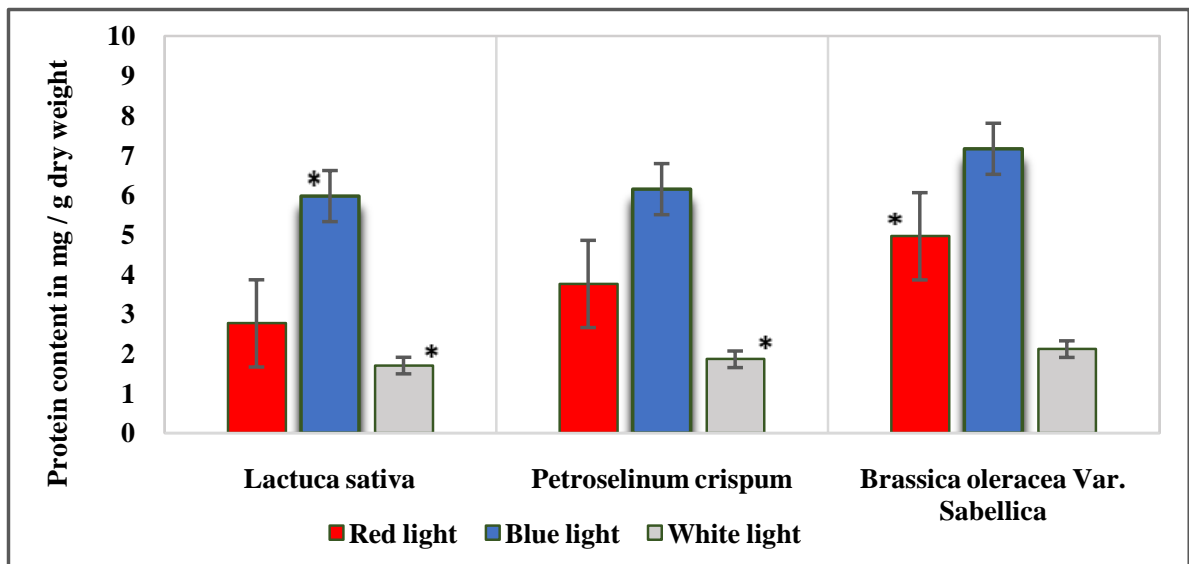

Figure 3 Effect of the light spectrum on the protein content of the studied plant species (Values indicated with asterisks are significantly different at $\mathrm{p} \leq 0.5$, each experiment was conducted in triplicates)

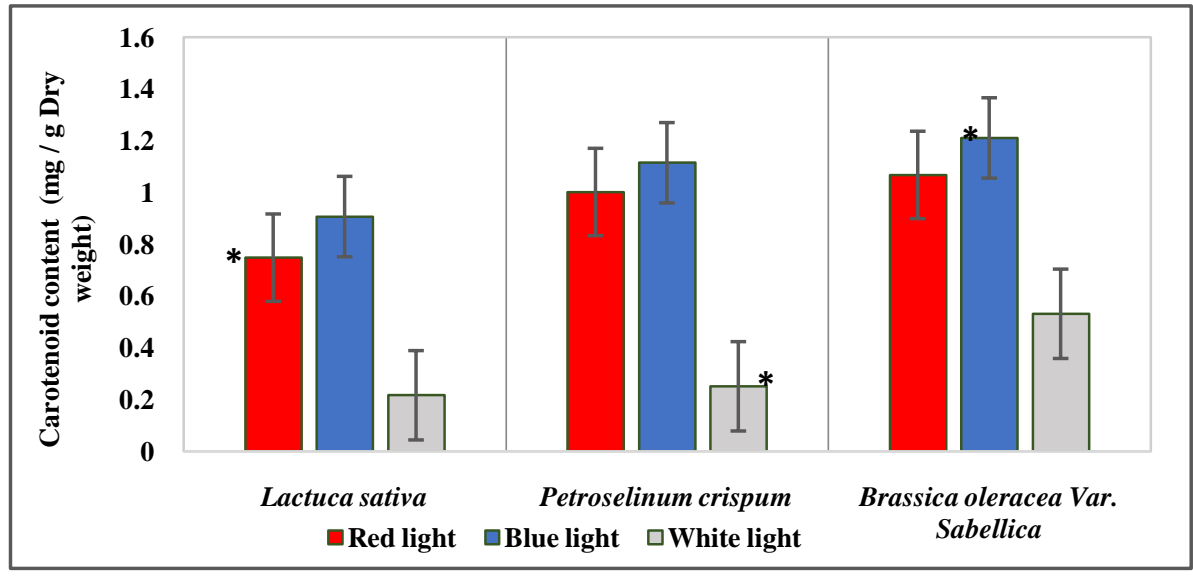

Figure 4 Effect of the light spectrum on the carotenoid content of the studied plant species (Values indicated with asterisks are significantly different at $\mathrm{p} \leq 0.5$, each experiment was conducted in triplicates)

\subsection{Effect of different} spectrum of light on the carotenoids content

The key role of carotenoids in living organisms is that they protect the body cells from the negative effects of free radicals. Another advantage of these biologically active substances is that they can accumulate in certain body tissues, thereby creating a protective effect. Accumulation of Lutein in the human retina reduced the risk of dystrophy, so-called macular spot. The great importance of carotenoids lies in their provitamin A activity. In this regard, the effect of the light spectrum on the accumulation of carotenoids in the selected plant species was studied in the current study.

Based on the presented data, it was reported that the blue light had a positive effect on the carotenoid content in all studied plant species. The leaves of Kale cabbage, grown under blue fluorescent lamps, contained significantly higher amounts of carotenoids than other plants. The positive effect of the red part of the spectrum on the accumulation of carotenoids in all studied species was also found (Figure 4).

The increasing demand for natural dietary supplements encourages the researcher and farmers to grow plants under artificial environmental conditions such as a greenhouse or growth chamber. These greenhouse or growth chambers 
used artificial/supplementary light spectrum (e.g., fluorescent, metal halide, or HPS lamps) to substitute natural light which has a significant effect on plant growth and photosynthesis. Various previous studies have been well reported the effect of different light spectrum on the rate of photosynthesis and the accumulations of various bioactive ingredients such as chlorophyll, carotenoid, ascorbic acid, and protein. Further, modification in the light conditions or type of light directly or indirectly affects the efficiency of carotenoid, ascorbic acid, and protein accumulation in the plants (Ouzounis et al., 2015; Bayat et al., 2018; Toldi et al., 2019).

\section{Conclusion}

Thus, the results of the current study suggested that Kale cabbage has the highest content of ascorbic acid, proteins, carotenoids, and this was followed by L. sativa and P. crispum. It was also reported that plants grown under the blue spectrum light contain the greatest amount of vitamin C, provitamin A, and proteins, which suggested that this light can be used for growing crops under greenhouse conditions.

\section{Acknowledgments}

The work is performed according to the Russian Government Program of Competitive Growth of Kazan Federal University.

\section{Conflict Of Interest}

Authors would hereby like to declare that there is no conflict of interests that could possibly arise.

\section{References}

Bayat L, Arab M, Aliniaeifard S, Seif M, Lastochkina O, Li T (2018) Effects of growth under different light spectra on the subsequent high light tolerance in rose plants. AoB PLANTS 10(5):ply052, https://doi.org/10.1093/aobpla/ply052.

Darko EHeydarizadeh P, Schoefs B, Sabzalian MR (2014) Photosynthesis under artificial light: the shift in primary and secondary metabolism.Philosophical Transactions of the Royal Society B: Biological Sciences 369 (1640): 20130243.

Houghton CA, Fassett RG, Coombes JS (2013) Sulforaphane: translational research from laboratory bench to clinic. Nutrition reviews 71(11): 709-726.

Kaulmann A, Jonville MC, Schneider YJ, Hoffmann L, Bohn T (2014) Carotenoids, polyphenols and micronutrient profiles of Brassica oleraceae and plumvarieties and their contribution to measures of totalantioxidant capacity. Food chemistry 155: 240-250.
Korus A, Lisiewska Z (2011) Effect of preliminary processing and method of preservation on the content of selected antioxidative compounds in kale (Brassica oleracea L. var. acephala) leaves. Food chemistry 129(1):149-154.

Lowry OH (2005) Protein Measurement with the Folin Phenol Reagent.The journal of Biological Chemistry, - 280- e25 - July 15 -.https://www.jbc.org/content/280/28/e25.full.

Lowry OH, Rosebrough NJ, Farr AL, Randall RJ (1951) Protein measurement with the Folin phenol reagent. Journal of Biological Chemistry 193: 265-275.

Meyer H, Bolarinwa A, Wolfram G, Linseisen J (2006) Bioavailability of apigenin from apiin-rich parsley in humans. Annals of Nutrition and Metabolism 50(3): 167-172.

Morrow RC (2008) LED Lighting in Horticulture. HortScience 43: $1947-1950$

Ouzounis T, Rosenqvist E, Ottosen CO (2015) Spectral Effects of Artificial Light on Plant Physiology and Secondary Metabolism: A Review. HortScience 50(8): 1128-1135. DOI: https://doi.org/10.21273/HORTSCI.50.8.1128.

Riabinina EI, Zotova EE, Vetrova EN, Ponomareva NI, Iliushina TN (2011) A new approach to assessing the antioxidant activity of plant materials in the study of the process of adrenaline autooxidation . Chemistry of vegetable raw materials 3: 117-121.

Sibgatullina GV, Khaertdinova LR, Gumerova EA, Akulov AN, KostiukovaIuA, Nikonorova NA, Rumiantsev NI (2011) Methods for determining the redox status of cultured plant cells.- Kazan: Kazan (Volga Region) Federal University: 43-46.53-57.

Sirota TV (2000) A method for determining the antioxidant activity of superoxide dismutase and chemical compounds. RF patent No. 2144674, 20.01.2000.

Toldi D, Gyugos M, Darkó É, Szalai G, Gulyás Z, Gierczik K, Székely A, Boldizsár Á, Galiba G, Müller M, Simon-Sarkadi L, Kocsy G (2019) Light intensity and spectrum affect metabolism of glutathione and amino acids at transcriptional level. PloS one 14(12), e0227271. https://doi.org/10.1371/journal.pone.0227271.

Walsh RP, Bartlett H, Eperjesi F (2015) Variation in carotenoid content of kale and other vegetables: A review of pre-and postharvest effects. Journal of Agricultural and Food Chemistry 63(44): 9677-9682. 